\title{
A Smart Farming System for Circular Agriculture ${ }^{\dagger}$
}

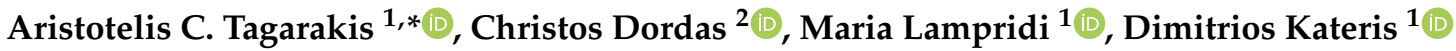 \\ and Dionysis Bochtis ${ }^{1}$
}

1 Centre for Research and Technology-Hellas (CERTH), Institute for Bio-Economy and Agri-Technology (IBO), 6th km Charilaou-Thermi Rd., GR 57001 Thessaloniki, Greece; m.lampridi@certh.gr (M.L.); d.kateris@certh.gr (D.K.); d.bochtis@certh.gr (D.B.)

2 School of Agriculture, Aristotle University of Thessaloniki, 54124 Thessaloniki, Greece; chdordas@agro.auth.gr

* Correspondence: a.tagarakis@certh.gr

+ Presented at the 13th EFITA International Conference, online, 25-26 May 2021.

check for updates

Citation: Tagarakis, A.C.; Dordas, C.; Lampridi, M.; Kateris, D.; Bochtis, D. A Smart Farming System for Circular Agriculture. Eng. Proc. 2021, 9, 10. https://doi.org/10.3390/engproc 2021009010

Academic Editors: Charisios Achillas and Dimitios Aidonis

Published: 24 November 2021

Publisher's Note: MDPI stays neutral with regard to jurisdictional claims in published maps and institutional affiliations.

Copyright: (C) 2021 by the authors. Licensee MDPI, Basel, Switzerland. This article is an open access article distributed under the terms and conditions of the Creative Commons Attribution (CC BY) license (https:// creativecommons.org/licenses/by/ $4.0 /)$.

\begin{abstract}
Agricultural activities such as fertilization and other crop management techniques contribute to greenhouse gas emissions and pollution; the cost of such activities is considerably high, with nitrogen pollution costing the EU up to 320 billion euros annually. In the present study, an integrated system has been proposed which utilizes smart farming tools and smart processing methodologies following the concept of a circular economy to reduce the impact of agricultural activity on climate change. Circular agriculture and precision farming together with the use of appropriate crop management tools may contribute to better resource use efficiency and sustainable agriculture.
\end{abstract}

Keywords: bioeconomy; circular agriculture; precision farming; smart farming; fertilization; resource use efficiency; sustainability

\section{Introduction}

Climate change is a major threat for the natural environment, and there is an imperative need to reduce greenhouse gas emissions. Agriculture contributes for about $10 \%$ of the greenhouse gas emissions and up to $95 \%$ of the emissions of ammonia in the European Union [1]. In order to reduce greenhouse gas emissions, new management approaches, such as circular agriculture, should be adapted, and new technologies that can minimize inputs for crop production must be developed and utilized. Circular agriculture is a modern agricultural management concept that promotes the reuse of all resources that can be used by the production system itself [2-4]. The utilization of such systems directly leads to the minimization of agricultural waste, while their application can reduce nutrient and water losses and emissions such as greenhouse gasses [4].

The latest advances in sensing and communication systems in agriculture have paved the way towards developing and following more efficient management schemes that account for the temporal and spatial variability of field and crop properties for timely and accurate field applications [5]. To be able to take advantage of these advanced sensing and management tools, the development of integrated systems with user-friendly interfaces is a prerequisite. As such, precision farming technologies can be utilized in conjunction with Internet of Things (IoT) sensing systems, in both crop and animal production processes, to feed the integrated system with real-time data $[4,6]$.

In this study, a holistic system for circular agriculture is proposed (BIOCIRCULAR) to be applied at small-medium vertical production dairy farms. Part of the system involves integrated soil fertility management which combines the use of appropriate amounts of organic and inorganic fertilizers together with green manure and good agronomic practices $[4,6,7]$. Preliminary results of the crop fertilization study for crops grown for feed (Zea mays L.), as part of the crop production sub-system, are also discussed. 


\section{System Requirements and Design}

The objective of the present study was to develop a precision field management and smart farming tool applied at every stage of the production chain. In order to properly design and develop such a tool, the system and user requirements were initially set. To that end, the procedures of the typical production dairy farms were divided into three main processes and analyzed, defining the inputs and outputs for each process step (Figure 1).

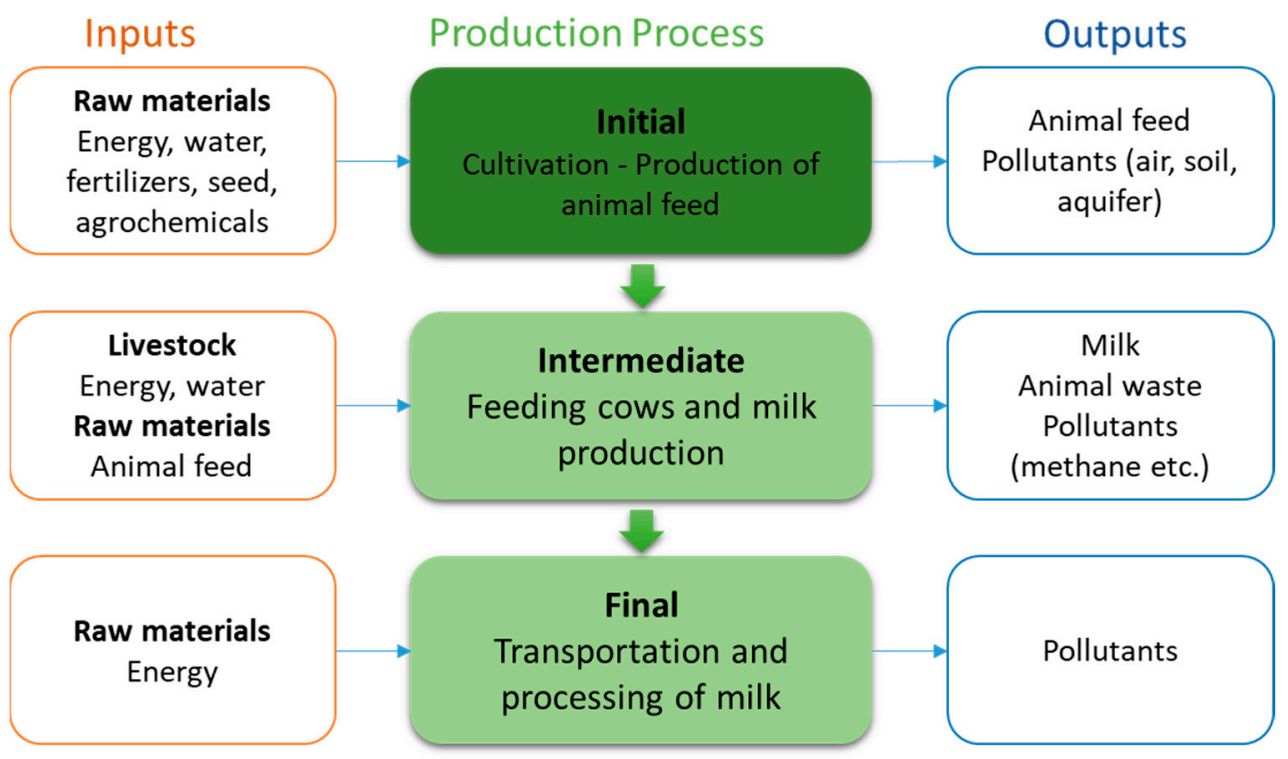

Figure 1. Production chain processes of small-medium vertical production dairy farms.

The system includes all procedures throughout the production chain and was therefore broken down into the basic sub-systems that are connected and interacting with a central circular precision management information system, which is the heart of the circular farm ecosystem (Figure 2). The sub-systems include: (a) crop production, (b) feed production, (c) animal production, and (d) animal waste management. Within the animal waste subsystem procedures, energy and organic fertilizer production are also included as biproducts to be used by the system itself (circular management). IoT and precision farming systems are involved in each sub-system to monitor and record the parameters needed for informed management.

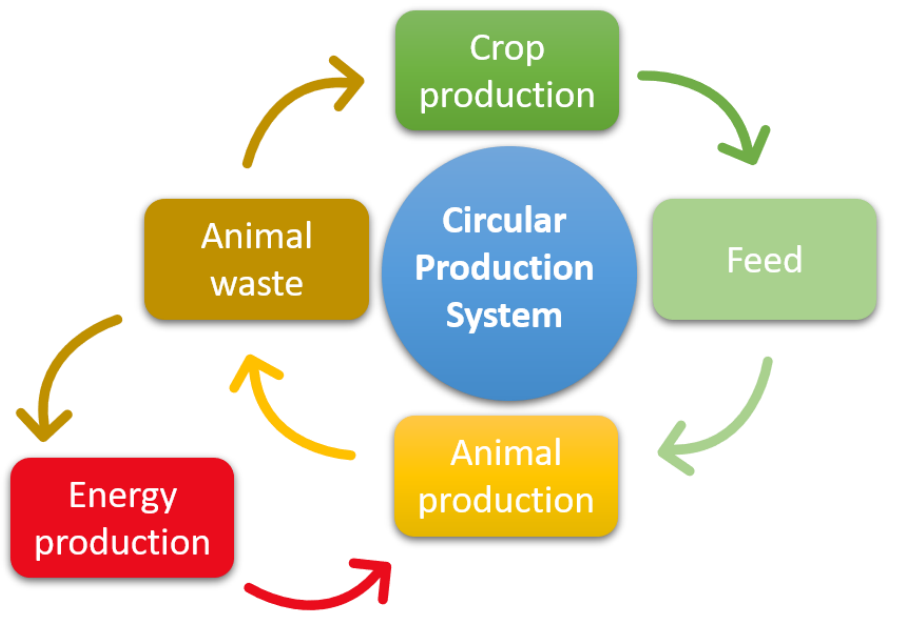

Figure 2. Circular production system. 


\section{The Platform}

The circular production system can receive and process all the information from each subsystem installed at every production level in the farm and provides the results in a simple and manageable form, supporting users to make appropriate decisions (Figure 3). Examples of input data include: (a) for the crop production sub-system-weather and soil moisture data from IoT, satellite and drone images, and crop and soil properties mapping proximal sensor data; (b) for the feed production sub-system-silage moisture and temperature data from IoT; (c) for the animal production sub-system-animal housing environmental conditions (temperature, humidity, $\mathrm{CO}_{2}$ and methane concentrations, etc.) monitored using IoT sensing systems, animal health status (temperature, activity, and behavior) using wireless IoT-enabled collars; (d) for the animal waste treatment subsystem-waste volume, waste water content and temperature, produced volume of biogas (if equipped with biogas production facilities), using IoT systems.

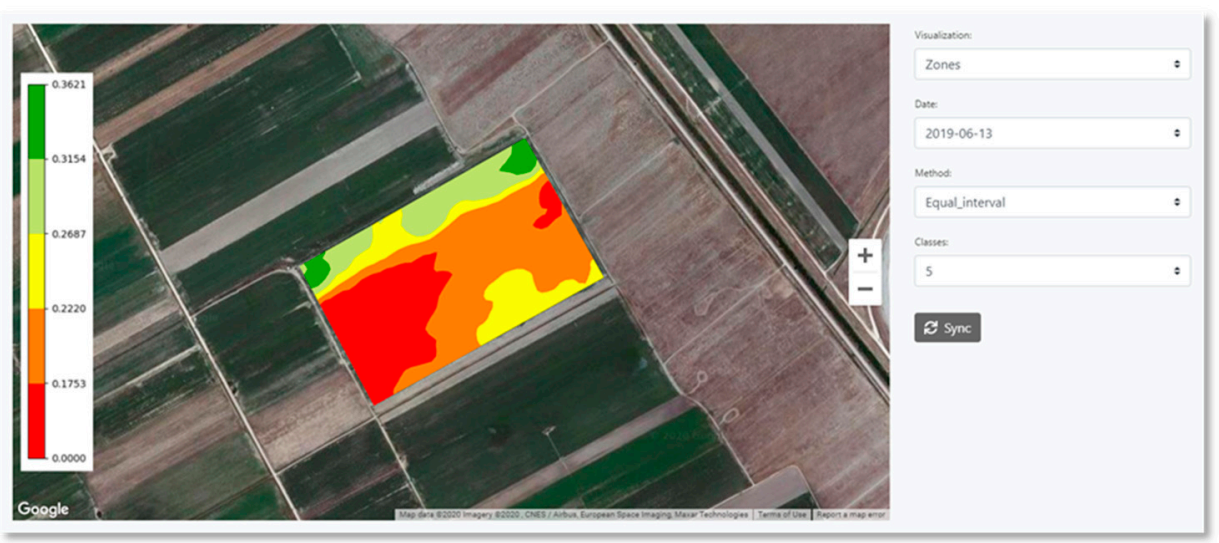

(a)

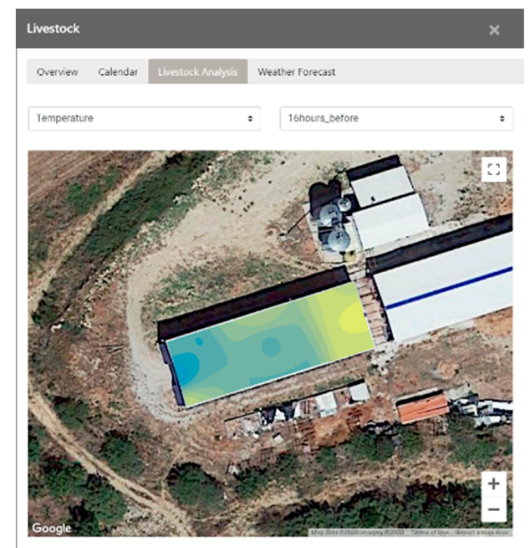

(b)

Figure 3. Some of the basic functions of the platform; (a) automated mapping of within-field crop properties and (b) mapping of environmental conditions inside the animal housing facilities.

\section{Crop Production Experiments}

Crop production is the initial production stage in a circular dairy system and consists of the first sub-system in BIOCIRCULAR. Within the scope of the project, field experiments were setup to study and develop management practices that may lead to increased production efficiency by minimizing the environmental footprint. In this work, the yield response of maize crop (Zea mays L.), grown for forage, to the application of different types of fertilizer (organic-manure and green manure; chemical fertilizers) was also investigated. Two maize hybrids were included in the study, namely Dekalb6777 and Pioneer1291. Five different fertilization treatments were tested: green manure (organic), manure (organic), conventional fertilizer (synthetic), slow-release fertilizer (synthetic), and control (no fertilizer applied).

The results showed that cattle manure can provide the nutrients in an available form that are essential for plant growth, while enhancing soil water-retention properties, leading to increased productivity of maize (Figure 4). Additionally, green manure with common vetch can provide adequate amounts of nitrogen, which is very important for maize. Therefore, efficient management of natural resources together with integrative crop management approaches can reduce inputs in crop production systems. 


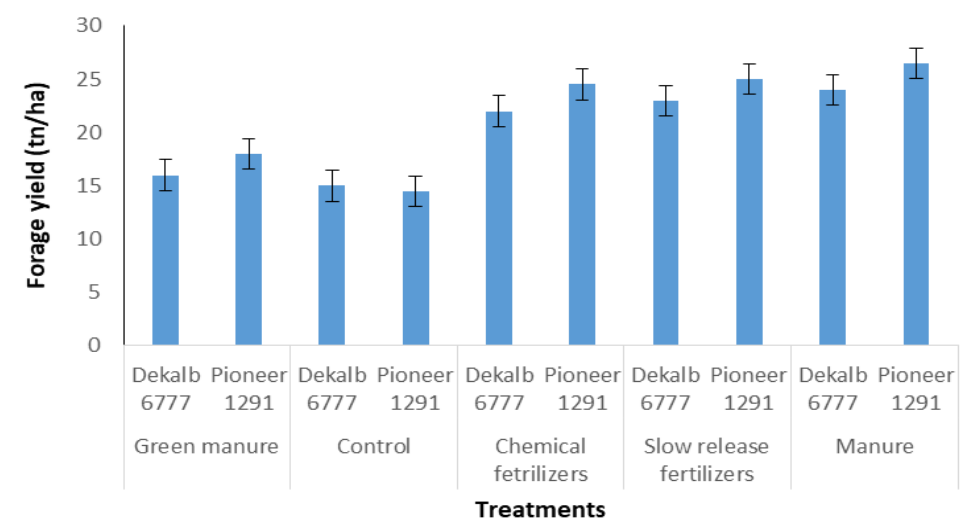

Figure 4. Forage yield of the different treatments that were tested with two maize hybrids during the 2019 growing season.

\section{Conclusions}

The benefits of using circular farming systems and digital technology are numerous. Implementing circular precision farming approaches may lead to sustainable development in dairy production, increasing efficiency, reducing inputs, and minimizing waste. More specifically, these systems enable monitoring of processes in the production stages, facilitating traceability towards producing certified products. In addition, the use of precision farming can increase the efficiency of the resources' utilization, by appropriately managing the spatial variability of fields. Furthermore, by supporting farmers in making optimal management decisions, it can increase the use efficiency of inputs, leading to their reduction, thus providing a financial benefit for the producers. Moreover, the circular characteristics of these systems minimize the production of animal waste and diminishes losses of nutrients and agrochemicals, thus reducing the environmental footprint.

The system presented in this study aims to constitute a dynamic, intelligent decision support system for circular agriculture that combines information from each available source throughout the whole production chain and supports farmers and farm managers in taking informed and timely decisions based on real-time data. Furthermore, the preliminary results from the crop fertilization experiment showed that using cattle manure as the sole source of fertilizer may lead to increased yields as compared to synthetic fertilizers. This result confirms the perspective of using animal waste in circular dairy production systems for increased sustainability.

Author Contributions: Conceptualization, A.C.T. and C.D.; methodology, A.C.T., C.D. and M.L.; validation, A.C.T., D.K. and D.B.; formal analysis, A.C.T. and C.D.; investigation, A.C.T., C.D. and M.L.; resources, A.C.T., C.D. and D.B.; data curation, A.C.T. and C.D.; writing-original draft preparation, A.C.T., C.D. and M.L.; writing-review and editing, A.C.T., D.K. and D.B.; visualization, A.C.T.; supervision, A.C.T., C.D. and D.B.; project administration, A.C.T., C.D. and D.B.; funding acquisition, A.C.T., C.D. and D.B. All authors have read and agreed to the published version of the manuscript.

Acknowledgments: This research has been supported by the European Union and Greek national funds through the Operational Programme Competitiveness, Entrepreneurship and Innovation, under the call RESEARCH-CREATE-INNOVATE (project code: T1EDK-03987) "BioCircular: Bioproduction System for Circular Precision Farming".

Conflicts of Interest: The authors declare no conflict of interest.

\section{References}

1. Eurostat Pocketbooks Agriculture, Forestry and Fishery Statistics; European Union: Luxembourg, 2013; ISBN 9789279330056.

2. von Braun, J. Bioeconomy-The global trend and its implications for sustainability and food security. Glob. Food Sec. 2018, 19, 81-83. [CrossRef] 
3. Kircher, M. Bioeconomy—Present status and future needs of industrial value chains. New Biotechnol. 2021, 60, 96-104. [CrossRef] [PubMed]

4. Rodias, E.; Aivazidou, E.; Achillas, C.; Aidonis, D.; Bochtis, D. Water-Energy-Nutrients Synergies in the Agrifood Sector: A Circular Economy Framework. Energies 2020, 14, 159. [CrossRef]

5. Anagnostis, A.; Tagarakis, A.C.; Kateris, D.; Moysiadis, V.; Sørensen, C.G.; Pearson, S.; Bochtis, D. Orchard mapping with deep learning semantic segmentation. Sensors 2021, 21, 3813. [CrossRef] [PubMed]

6. Lampridi, M.G.; Sørensen, C.G.; Bochtis, D. Agricultural Sustainability: A Review of Concepts and Methods. Sustainability 2019, 11, 5120. [CrossRef]

7. Dordas, C.A.; Lithourgidis, A.S.; Matsi, T.; Barbayiannis, N. Application of liquid cattle manure and inorganic fertilizers affect dry matter, nitrogen accumulation, and partitioning in maize. Nutr. Cycl. Agroecosyst. 2007, 80, 283-296. [CrossRef] 